\title{
Directional CSMA/CA Protocol with Spatial Reuse for mmWave Wireless Networks
}

\author{
Michelle X. Gong, Dmitry Akhmetov, Roy Want \\ Intel Labs, Intel Corporation \\ Santa Clara, CA 95054-1549 \\ Email: \{michelle.x.gong, dmitry.akhmetov, roy.want\}@intel.com
}

\author{
Shiwen Mao \\ Department of ECE, Auburn University \\ Auburn, AL 36849-5201 \\ Email: smao@ieee.org
}

\begin{abstract}
In recent years, the millimeter wave (mmWave) technology has gained considerable interest due to the huge unlicensed bandwidth (i.e., up to $7 \mathrm{GHz}$ ) available in the $60 \mathrm{GHz}$ band in most part of world. In this paper, we investigate the problem of medium access control (MAC) in mmWave wireless networks, within which directional antennas are used to combat the high path loss incurred in the $60 \mathrm{GHz}$ band. We extend a directional CSMA/CA protocol presented in our prior work by exploiting spatial reuse. The proposed protocol adopts virtual carrier sensing and allows non-interfering links to communicate simultaneously. We present a performance analysis as well as simulations to evaluate the proposed protocol. Our results show that the directional MAC with spatial reuse can achieve considerable performance improvements over the 802.11 MAC and the protocol proposed in our prior work. It introduces low protocol overhead and has robust performance even when the network is heavily congested.
\end{abstract}

\section{INTRODUCTION}

In recent years, the millimeter wave (mmWave) technology has gained considerable interest from academia, industry, and standards bodies. This is due to the huge unlicensed bandwidth (i.e., up to $7 \mathrm{GHz}$ ) that is available in the $60 \mathrm{GHz}$ band in most part of world. With this massive unlicensed bandwidth, many bandwidth-demanding new applications can be easily supported in mmWave wireless local area networks (WLAN).

The $60 \mathrm{GHz}$ regulation allows much higher effective isotropic radiated power (EIRP) compared to other existing WLANs and wireless personal area networks (WPANs). Such high EIRP is required since signals in the $57-64 \mathrm{GHz}$ region are subject to a resonance of the oxygen molecule and are thus severely attenuated. The propagation attenuation of $60 \mathrm{GHz}$ signals in free space is $22 \mathrm{~dB}$ higher than that of $5 \mathrm{GHz}$ signals. $60 \mathrm{GHz}$ signals also suffer from high attenuation loss due to obstacles. For instance, a human body introduces at least $15 \mathrm{~dB}$ loss to $60 \mathrm{GHz}$ signals compared to only $5 \mathrm{~dB}$ loss to $5 \mathrm{GHz}$ signals. Therefore, directional antennas, such as phased antenna arrays, are required to overcome the high propagation loss. Directional transmissions should be explicitly considered in the design of MAC protocol for mmWave wireless networks.

In $60 \mathrm{GHz}$ band systems, beamforming, on both the receive side and the transmit side, is used to improve signal quality at the receiver. As a result of directional listening and transmitting, the signal strength could be very low at third party stations that are not involved in the current exchange, making it difficult to perform carrier sense. This is often referred to as the deafness problem, making traditional carrier sensing mechanisms inadequate for medium access control. New mechanisms are needed to ensure that deferral by third party stations is effective in this environment. On the other hand, such reduced interference to neighboring links can be exploited for spatial reuse. It is possible to schedule concurrent transmissions at multiple links without interfering each other, so as to improve the throughput performance of the system.

In our prior work [1], we propose a directional Carrier Sense Multiple Access with Collision Avoidance (CSMA/CA)-based medium access protocol that is tailored for $60 \mathrm{GHz}$ wireless networks. Instead of relying on physical carrier sensing, the proposed protocol adopts virtual carrier sensing and relies on a central coordinator (e.g., the AP) to distribute network allocation vector (NAV) information. However, the protocol proposed in [1] does not take advantage of spatial reuse gain in a wireless network with directional transmissions. Due to the small interfering area of highly directional antennas, simultaneous communications in the same frequency channel and in the same physical space are highly possible [2]. Therefore, a MAC protocol that takes advantage of the spatial reuse gain could increase the aggregate network capacity.

In this paper, we propose an enhanced directional CSMA/CA protocol that retains the advantages of the previously proposed protocol in [1] and, moreover, enables spatial reuse in mmWave WLANs. With the enhancement, the AP first schedules a link to transmit data and schedules interference measurement at each peer link. Based on measurements, the AP assigns non-interfering peer links into the same group. Since they do not interfere with each other while operating in the directional mode, peer links in the same group can be scheduled to transmit simultaneously. Such concurrent transmissions will improve the system throughput performance.

We present an analysis based on a Markov Chain model of the directional system [3], which can be used to derive the system saturation throughput and the optimal transmission probability for a station (STA). We also implement the proposed protocol using OPNET Modeler and evaluate its performance with simulations. Both analytical and simulation results demonstrate that the proposed directional CSMA/CA MAC protocol with spatial reuse achieves considerable throughput gains over the 802.11 MAC and the directional CSMA/CA MAC protocol proposed in [1]. 
The remainder of this paper is organized as follows. We first discuss related work in Section II. We then introduce the system model and the proposed protocol in Section III. Our analysis and simulation study are presented in Sections IV and V, respectively. Section VI concludes this paper.

\section{RELATED WORK}

Currently, several standards have been or are being defined to achieve multi-gigabit rates for $60 \mathrm{GHz}$ wireless networks, such as ECMA-387 [4] and IEEE 802.15.3c [5]. Both standards focus on using Time Division Multiple Access (TDMA) for data communications. Existing MAC protocols recently proposed for $60 \mathrm{GHz}$ networks [6], [7] are also based on TDMA. Because data traffic is bursty, the required medium time is often highly unpredictable. A TDMA-based MAC protocol may cause either high overhead for on-the-fly medium reservation, or under- or over-allocated medium time for individual users. Furthermore, because scheduling is computationally intensive and should be executed in real time, it is challenging to implement such an AP on a mobile station.

Contention-based MAC protocols, such as CSMA/CA, work well with bursty traffic and operate robustly in unlicensed bands [3]. However, CSMA/CA does not work well with directional antennas due to impaired carrier sensing. Under CSMA/CA, stations (STAs) that see a busy medium compete for access to the medium by waiting a random number of slot times before the next attempt for transmission. Usually the STA with the smallest random number will gain access to the medium. The other STAs will detect the transmission through a carrier sense mechanism and defer their attempts for channel access until the medium becomes idle again.

Many contention-based MAC protocols that support directional antennas have been proposed for mobile ad hoc networks or wireless mesh networks [8]-[12]. A short survey can be found in [13]. Most of the schemes rely on RTS and/or CTS to distribute the Network Allocation Vector (NAV), which contains duration information of the subsequent transmission(s). However, most existing directional MAC protocols either are based on assumptions that do not apply to the $60 \mathrm{GHz}$ band, or introduce too much overhead when adopted for $60 \mathrm{GHz}$. For instance, [8] proposes that a transmitter senses the medium for Data plus SIFS time and transmit $2 M$ directional RTS (DRTS) frames before transmitting a data frame, where $M$ is the number of sectors. This approach introduces considerable overhead for each data transmission. Further, it does not work well when there is bi-directional traffic, meaning a node is both a transmitter and a receiver. Section III-A elaborates on why other existing directional MAC protocols cannot be directly applied in the $60 \mathrm{GHz}$ band.

\section{PROTOCOL DESCRIPTION}

\section{A. System Model}

We consider a wireless LAN as illustrated in Fig. 1. In such a network, there is one AP that coordinates medium access for multiple mobile STAs. The AP also provides basic timing and manages membership of the network. STAs can communicate with the AP or they can communicate directly with each other without having to bridge data through the AP.

A unique challenge in the design of $60 \mathrm{GHz}$ wireless networks arises from the requirement for high antenna gain at both the transmitter and receiver. Before two stations can finish beam-forming training with each other, neither one can achieve proper beam-formed transmission or reception. Therefore, a low-rate modulation and coding scheme (MCS) needs to be defined to address the case when only one end of the link has high beam-forming gain. To this end, we assume a range optimized MCS, i.e. MCS0, which has a receiver sensitivity about $12 \mathrm{~dB}$ higher than that of a data rate optimized MCS that offers data rates higher than $1 \mathrm{Gbps}$. Many existing directional contention-based MAC protocols assume that normal data rate can be maintained even when only one end of the link uses directional antennas. This assumption is not valid anymore in the $60 \mathrm{GHz}$ band. In $60 \mathrm{GHz}, \mathrm{MCS} 0$ has to be used for beamforming training and, moreover, for transmissions when only one end of the link has high antenna gain.

Furthermore, existing directional MAC protocols assume that beam-training or the so-called beam-locking can be performed on a single received packet. While this might be true for MIMO systems operating in lower frequency bands (e.g., $2.4 \mathrm{GHz}$ or $5 \mathrm{GHz}$ ), beam-forming training at $60 \mathrm{GHz}$ with phased arrays is much more challenging and requires multiple iterations. In a $60 \mathrm{GHz}$ system, if an STA needs to transmit a frame (e.g., an RTS) in an omni-directional fashion, it needs to perform a sector sweep to transmit multiple copies of the RTS frame in different directions or sectors. During the RTS transmission, the STA may miss any transmission intended for it from other STAs. Thus, it would be nontrivial to solve the deafness problem in the $60 \mathrm{GHz}$ band with existing contentionbased directional MAC protocols.

\section{B. Directional CSMA/CA Protocol with Spatial Reuse}

We describe the directional CSMA/CA MAC with spatial reuse in this section. With this protocol, before associating with the AP, STAs first perform beam-forming training for transmission and reception, such that both the transmitting and the receiving antennas can provide beam-forming gain. After an STA completes beam-forming training, it always beam-forms towards the AP in its idle mode (i.e., waiting to receive), meaning there is high receive antenna gain. While idle, the AP receives in its omni mode, meaning that there is no significant receive antenna gain. Because an STA is always beam-formed towards the AP before any data transmission or reception and the AP coordinates the transmission within a WLAN, the deafness problem is thus easily solved.

To describe the protocol operation, we consider a typical scenario shown in Fig. 1. In this scenario, STA1 and STA2, STA3 and STA4, STA3 and STA5 have set up peer-to-peer connections respectively. In the idle mode, all stations are beam-formed towards the AP. The AP schedules interference measurement when a peer link is being established. For instance, the AP schedules STA1 and STA2 to transmit and receive over the peer link, while all other STAs stay in direc- 


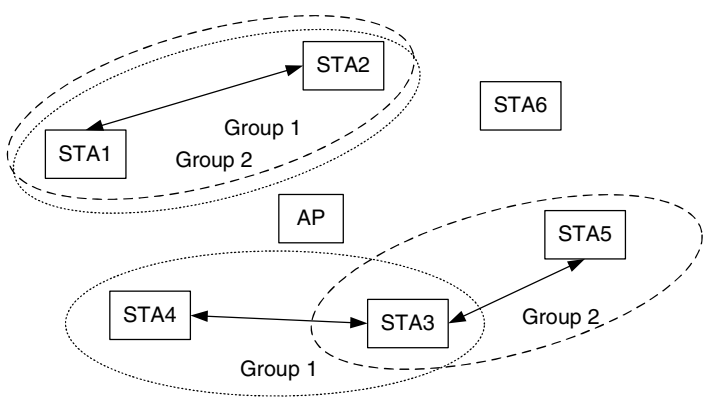

Fig. 1. A WLAN network consisting of one AP and six STAs. Two groups are formed: Group 1 with links (STA1-STA2) and (STA3-STA4), and Group 2 with links (STA1-STA2) and (STA3-STA5).

tional receive mode to measure the noise floor. Based on the interference measurement, the AP assigns non-interfering peer links into the same group. Note that because the interference measurement is performed during the link measurement phase of the peer link establishment, there is no additional overhead. As shown in Fig. 1, peer link (STA1-STA2) and peer link (STA3-STA4) are assigned to Group 1, whereas peer link (STA1-STA2) and peer link (STA3-STA5) are assigned to Group 2. Because peer links in the same group do not interfere with each other in directional transmission and receiving mode, they can communicate simultaneously.

The operation of the proposed protocol is illustrated in Fig. 2. Before STA1 can communicate with STA2 directly, it transmits a Target Request To Send (TRTS) to the AP. The TRTS contains three addresses: Receive Address (i.e. AP), Transmit Address (i.e. STA1), and Target Address (i.e. STA2). Upon receiving the TRTS, the AP will transmit a Target Clear To Send (TCTS) message in omni mode. This ensures that all associated STAs can receive it. The TCTS contains the following fields: Receive Address (i.e. the broadcast address), Transmit Address (i.e. AP), Target Address (i.e. STA2), a Group ID field, and a transmission priority field. In this case, the Group ID is set to 1, indicating that all peer links that belong to Group 1 may transmit in the following Transmission Opportunity (TXOP). If the transmission priority field is set to 1 , the peer STA with higher MAC address transmits first. If the transmission priority field is set to 0 , the peer STA with lower MAC address transmits first. If an STA with lower priority senses the medium idle PIFS (Point Inter-frame Space) after receiving the TCTS, it may start transmission to its peer STA in the same group. Both TRTS and TCTS indicate the duration of the TXOP and they are transmitted using MCSO because only one end of the link has beam-forming gain. If the AP does not receive the TRTS due to either channel error or a collision on TRTS, STA1 will not receive a TCTS after transmitting a TRTS. Thus, STA1 assumes that a collision has occurred and starts an exponential backoff procedure as defined in [14].

After receiving the TCTS and recognizing that to the TCTS allows peer links in Group 1 to communicate simultaneously, STA1 and STA2 steer its beam towards each other while STA3 and STA4 steer its beam towards each other. Upon receiving a TCTS with its own address as the Target Address, STA2

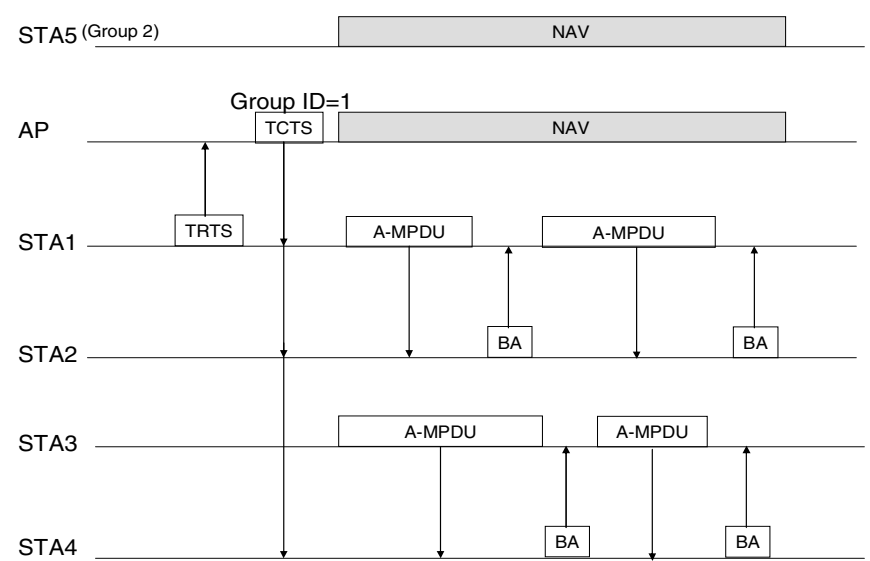

Fig. 2. Operation of the directional CSMA/CA MAC with spatial reuse.

should wait for STA1 to transmit first. If PIFS after receiving the TCTS frame, an STA senses the medium free, it can starts its data transmission towards its peer STA. The TRTS/TCTS exchange sets up a transmission opportunity (TXOP) in the network. Within the TXOP, STA1 can transmit one or more Aggregate MAC Protocol Data Units (A-MPDUs) to STA2 at a high data rate. Up to 64 MPDUs may be aggregated in one A-MPDU, which has a maximum size limit of 64K [14]. Upon receiving an A-MPDU, STA2 replies with a Block ACK (BA) that identifies which MPDUs in the A-MPDU have been received successfully. Other stations that do not belong to Group 1, such as STA5, learn from the TCTS that there will be an on-going transmission and thus set their NAVs for the duration of the TXOP indicated in the TCTS. Note that STAs transmitting in the TXOP have to obey the TXOP duration and should not transmit beyond the TXOP boundary that was defined by the TRTS/TCTS exchange.

\section{Discussions}

Even though the proposed directional MAC protocol bears similarities with 802.11 DCF [14], there are a few important differences that are worth noting. First, DCF is a distributed MAC protocol, meaning any STA can transmit an RTS to any other STA. On the other hand, the proposed directional CSMA/CA protocol is a centralized protocol. Before any data transmission, an STA must transmit a TRTS to the AP to reserve medium time. Due to antenna directionality, STAs adopting a distributed MAC protocol need to continuously track all neighboring STAs, which can cause prohibitively high communication overhead and high implementation complexity.

Second, to support directionality, some of the parameters in the proposed protocol are different from those defined in 802.11 DCF. For instance, aSlotTime is an important parameter in both DCF and our protocol and it is set to the time needed for any station to detect a transmission from any other station. In 802.11 DCF, aSlotTime is set to:

$$
\begin{aligned}
& \text { aSlotTime } \\
= & \text { aCCATime }+ \text { aRxTxTurnaroundTime }+ \\
& \text { aAirPropagationTime }+ \text { aM ACProcessingDelay, }
\end{aligned}
$$




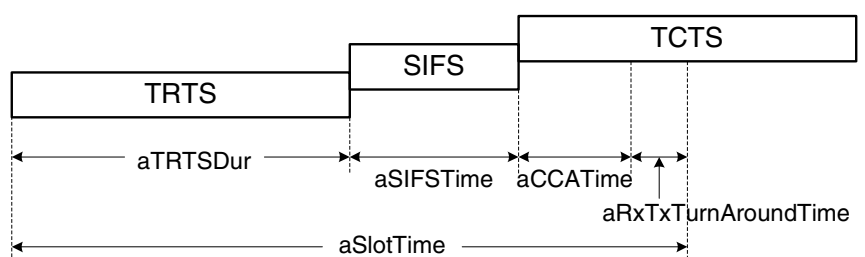

Fig. 3. Illustration of aSlotTime in the directional CSMA/CA.

whereas in our protocol, aSlotTime is set to:

$$
\begin{aligned}
& \text { aSlotTime } \\
= & \text { aTRTSDur + aSIFSTime + aCCATime }+ \\
& \text { aRxTxTurnAroundTime, }
\end{aligned}
$$

where aTRTSDur is the duration of a TRTS frame, including the PHY preamble, the PHY header and the TRTS frame body. aSIFSTime is a short inter-frame time between receiving a packet and sending out an acknowledgment. aCCATime is the time that a receiver needs to determine whether a valid packet is on the medium. aRxTxTurnaroundTime is the time that a half-duplex station needs to switch from $\mathrm{Rx}$ mode to Tx mode. Because all STAs are beam-formed towards the AP and the width of the beam generated by an antenna array is narrow, most other STAs won't be able to receive the TRTS sent from the source STA. Therefore, for a third-party STA to detect an on-going transmission, virtual carrier sensing has to be used and thus aSlotTime needs to include aTRTSDur and aSIFSTime. The definition of aSlotTime in the proposed protocol is illustrated in Fig. 3.

Last but not least, 802.11 DCF was designed mainly for omni-directional transmissions and it suffers from various problems, including the deafness problem, when being used with directional antennas. Our proposed protocol is designed specifically for directional transmissions in the $60 \mathrm{GHz}$ band and it addresses the deafness problem.

\section{Performance Analysis}

In this section, we present an analytical study of the proposed directional MAC protocol. We derive the saturation throughput of the proposed protocol, which is defined as the throughput level achieved at the top of the MAC layer when all nodes in the systems are continuously loaded.

It is assumed that stations use MAC frame aggregation schemes, such as A-MPDU, and may make multiple transmissions in one TXOP. When TXOP is utilized, a station contends once to transmit TRTS. Upon successful reception of a TCTS that allows a station to transmit, the station can transmit as many A-MPDU as the TXOP duration permits, provided that the last BA can be received within the TXOP duration.

We follow the assumptions made in [3] and adopt the same 2-D Markov chain model for the proposed MAC protocol. In the Markov chain mode, each state is represented by $\{s(t), b(t)\}$, where $s(t)$ is defined to be the stochastic process representing the backoff stage $\{0, \cdots, m\}$ of the station at time $t$ and $b(t)$ is the stochastic process representing the backoff time counter for a given station. The maximum backoff stage, i.e., $m$, takes the value such that $C W_{\max }=2^{m} C W_{\min }$, where $C W_{\max }$ is the maximum contention window and $C W_{\min }$ is the minimum contention window.

Let $S$ be the normalized system throughput, defined as the fraction of time the channel is used to successfully transmit payload bits. $S$ can be expressed as the average number of payload bits transmitted in a TXOP divided by the average length of a TXOP. Based on the 2-D Markov chain model, we derive the system saturation throughput as:

$$
\begin{aligned}
S= & P_{A P_{-} S T A} \frac{P_{s} P_{t r} \sum_{j=1}^{N} \mathrm{E}\left[P_{j}\right]}{\left(1-P_{t r}\right) \sigma+P_{t r} P_{s} T_{s}+P_{t r}\left(1-P_{s}\right) T_{c}}+ \\
& P_{S T A \_S T A} \frac{P_{s} P_{t r} \sum_{j=1}^{M} \sum_{i=1}^{N_{j}} \mathrm{E}\left[P_{i j}\right]}{\left(1-P_{t r}\right) \sigma+P_{t r} P_{s} T_{s}+P_{t r}\left(1-P_{s}\right) T_{c}},
\end{aligned}
$$

where

$$
\left\{\begin{array}{l}
T_{s}=\sigma+a T C T S D u r+T X O P \\
T_{c}=\sigma \\
P_{t r}=1-(1-\tau)^{2} \\
P_{s}=\frac{n \tau(1-\tau)^{n-1}}{1-(1-\tau)^{n}} .
\end{array}\right.
$$

In (1) and (2), $P_{A P \_S T A}$ is the probability that an AP/STA pair wins the contention, $P_{S T A_{-} S T A}$ is the probability that an STA/STA pair wins the contention, $M$ is the number of peer links in one group, $T_{s}$ is the average time consumed by a successful TXOP, $T_{c}$ is the average medium time a collision consumes, $\sigma$ is the duration of a time slot, aTCTSDur is the transmission duration of the TCTS frame, $\tau$ is the probability that a station transmits in a randomly chosen time slot, $P_{s}$ is the probability that a TXOP is successfully set up, and $P_{t r}$ is the probability that there is at least one transmission in the slot time. $M$ is the number of peer links that can operate simultaneously in one group. The sum $\sum_{j=1}^{M} \sum_{i=1}^{N_{j}} \mathrm{E}\left[P_{i j}\right]$ is the combined average payload size of A-MPDUs that are transmitted over $M$ peer links in the TXOP.

Equation (1) can be rearranged as follows:

$S=\frac{1}{2 n} \frac{(n-1) \sum_{i=1}^{N} \mathrm{E}\left[P_{i}\right]+(n+1) \sum_{j=1}^{M} \sum_{i=1}^{N_{j}} \mathrm{E}\left[P_{i j}\right]}{T_{s}-T_{c}+\left[T_{c}-(1-\tau)^{n}\left(T_{c}-\sigma\right)\right] /\left[n \tau(1-\tau)^{n-1}\right]}$.

Conditioned on $\tau \ll 1$, we have $\tau \approx\left[n \sqrt{T_{c} /(2 \sigma)}\right]^{-1}$ [3].

In Fig. 4, we plot the relation between the optimal saturation throughput and the number of stations in the WLAN. When the transmission probability is small, i.e., $\tau \ll 1$, the throughput degradation is small with an increase in the number of stations.

\section{Simulation Study}

We evaluate the performance of the proposed directional MAC protocol with OPNET simulations. Our simulation uses a typical WLAN topology with one AP and five STAs in the network, as illustrated in Fig. 1. We assume that STA1 always has data to transmit to STA2, while STA4 and STA5 always have data to transmit to STA3. All the traffic sessions start at Second 2 of the simulation. The simulation parameters and the values are given in Table I. The simulation results are presented in Fig. 5. 


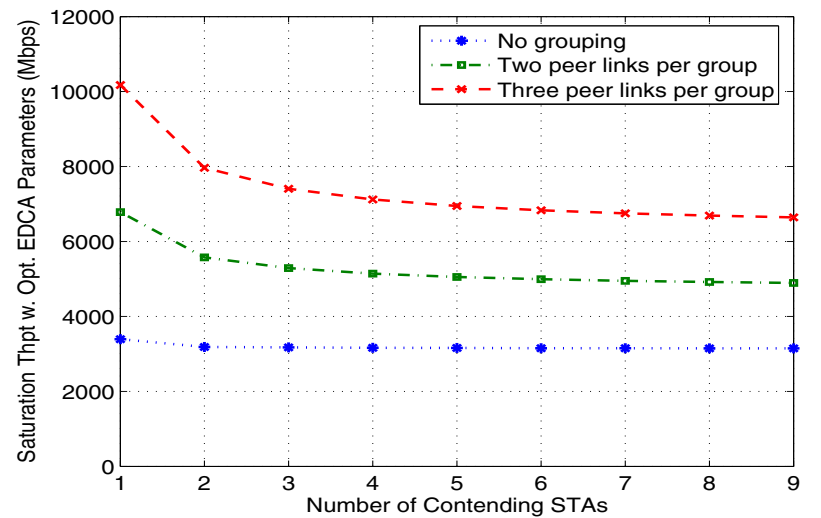

Fig. 4. Optimal saturation throughput vs. number of STAs in the WLAN.

TABLE I

Simulation PARAMETERS

\begin{tabular}{|l|l||l|l|}
\hline Parameter & Value & Parameter & Value \\
\hline \hline MCS0 Rate (Mbps) & 25 & aSlotTime (us) & 20 \\
\hline Data Rate (Mbps) & 4,063 & aSIFSTime (us) & 2 \\
\hline ACK Rate (Mbps) & 1,384 & TXOP duration (us) & 500 \\
\hline A-MPDU size (byte) & 65,536 & MCS0 preamble (us) & 3.75 \\
\hline TRTS (byte) & 26 & Data preamble (us) & 1.75 \\
\hline TCTS (byte) & 26 & Default CWmin & 15 \\
\hline BA size (byte) & 32 & Default CWmax & 1,023 \\
\hline
\end{tabular}

As shown in Fig. 5, the saturation throughput achieved by the directional CSMA/CA protocol proposed in [1] is about 3.5 Gbps in the steady state, which is $37 \%$ lower than that of the 802.11 protocol. On the other hand, the saturation throughput achieved by the directional CSMA/CA MAC with spatial reuse is about $5.2 \mathrm{Gbps}$, which is about $47 \%$ higher than that of the 802.11 protocol. It is almost twice higher than that of the directional CSMA/CA protocol without spatial reuse.

The directional CSMA/CA with spatial reuse protocol naturally inherits a main benefit of the 802.11 MAC, which is spatial reuse enabled by the highly directional data transmissions. By avoiding the deafness problem, the directional CSMA/CA with spatial reuse protocol significantly reduces the number of collisions within WLAN. There is also an additional gain provided by shortened channel access time in non interfering groups. While one peer link has finished contention and started a TXOP, another peer link in the same group may still be performing backoff. Reception of TCTS frame indicating transmission in the same non-interfering group enables data transmission for all peer links in this group.

\section{CONCLUSION}

We proposed and evaluated an extension to a directional CSMA/CA MAC protocol presented in our prior work. The extension exploits spatial reuse in mmWave networks with directional antennas. We show that it does not suffer from the deafness problem, and introduces a small overhead. Through OPNET simulations, we find that the proposed protocol achieves significantly higher throughput than the 802.11 MAC and the directional CSMA/CA protocol presented in our prior work. Both our analysis and simulation results demonstrate

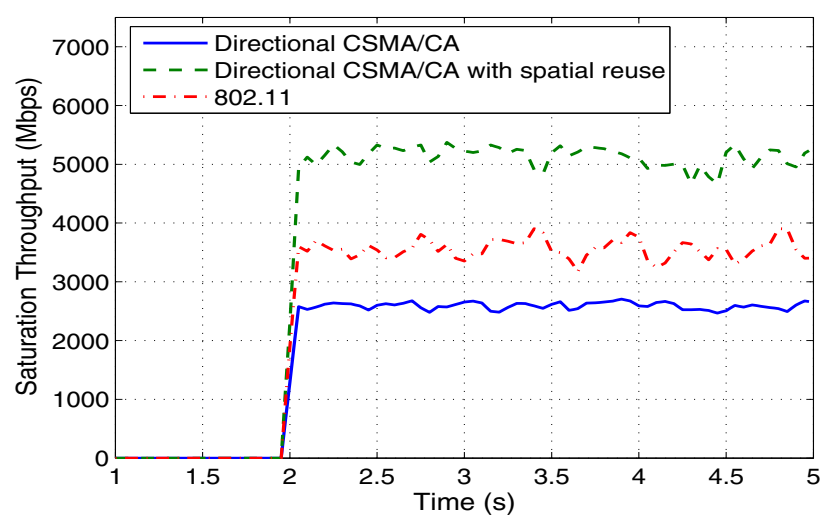

Fig. 5. Aggregate saturation throughput for the three schemes.

the high MAC efficiency achieved by the proposed directional MAC protocol for mmWave networks.

\section{ACKNOWLEDGMENT}

Shiwen Mao's research is supported in part by the US National Science Foundation (NSF) under Grants ECCS0802113, IIP-1032002, and through the Wireless Internet Center for Advanced Technology (WICAT) at Auburn University (under NSF Grant IIP-0738088).

\section{REFERENCES}

[1] M. Gong, R. Stacey, A. Dmitry, and S. Mao, "A directional CSMA/CA protocol for mmWave wireless PANs," in Proc. IEEE WCNC'10, Sydney, Australia, Apr. 2010, pp.1-6.

[2] R. Mudumbai, S. Singh and U. Madhow, "Medium access control for $60 \mathrm{GHz}$ outdoor mesh networks with highly directional links," in Proc. IEEE INFOCOM'09, Rio de Janeiro, Apr. 2009, Brazil, pp.2871-2875.

[3] G. Bianchi, "Performance analysis of the IEEE 802.11 Distributed Coordination Function," IEEE J. Sel. Areas Commun., vol.18, no.3, pp.535-547, Mar. 2000.

[4] ECMA TC48, ECMA standard 387, "High Rate 60GHz PHY, MAC and HDMI PAL," Dec. 2008.

[5] IEEE 802.15 WPAN Task Group, "3c millimeter wave alternative PHY (TG3c)," [online] Available: http://www.ieee802.org/15/pub/TG3c.html.

[6] X. An and R. Hekmat, "Directional MAC protocol for millimeter wave based wireless personal area networks," in Proc. IEEE VTC-Spring'08, Singapore, May 2008, pp.1636-1640.

[7] C.-W. Pyo, F. Kojima, J. Wang, H. Harada, and S. Kato, "MAC enhancement for high speed communications in the $802.15 .3 \mathrm{c} \mathrm{mmWave}$ WPAN," Springer Wireless Pers. Commun., vol.51, pp.825-841, 2009.

[8] E. Shihab, L. Cai and J. Pan, "A distributed, asynchronous directionalto-directional MAC protocol for wireless ad hoc networks," IEEE Trans. Veh. Technol., vol.58, no.9, pp.5124-5134, Nov. 2009.

[9] M. Takai, J. Martin, A. Ren, and R. Bagrodia, "Directional virtual carrier sensing for directional antennas in mobile ad hoc networks," in Proc. ACM MobiHoc'02, Lausanne, Switzerland, June 2002, pp.183-193.

[10] J. Hsu and I. Rubin, "Performance analysis of directional CSMA/CA MAC protocol in mobile ad hoc networks," in Proc. IEEE ICC'06, Istanbul, Turkey, June 2006, pp.3657-3662.

[11] Y.B. Ko, V. Shankarkumar, and N.H. Vaidya, "Medium access control protocols using directional antennas in ad hoc networks," in Proc. IEEE INFOCOM'O0, Tel Aviv, Israel, Mar. 2000, pp.13-21.

[12] T. Korakis, G. Jakllari, and L. Tassiulas, "A MAC protocol for full exploitation of directional antennas in ad-hoc wireless networks," in Proc. ACM MobiHoc'03, Annapolis, MD, June 2003, pp.98-107.

[13] M. Gong, S. Midkiff, and S. Mao, "MAC protocols for wireless mesh networks," in Wireless Mesh Networking: Architectures, Protocols and Standards, Y. Zhang, J. Luo, and H. Hu (Editors), pp.147-182. New York, NY: Auerbach Publications, 2006.

[14] IEEE standard 802.11, "Wireless LAN Medium Access Control (MAC) and Physical Layer (PHY) specification," 2007. 